\title{
Photocurable polymethacrylate-silica nanocomposites: correlation between dispersion stability, curing kinetics, morphology and properties
}

\author{
Mariola Sadej $^{1}$ - Hubert Gojzewski ${ }^{2,3} \cdot$ Ewa Andrzejewska $^{1}$
}

Received: 9 October 2015 / Accepted: 16 May 2016 / Published online: 24 May 2016

(C) The Author(s) 2016. This article is published with open access at Springerlink.com

\begin{abstract}
A comprehensive study of model systems based on poly(ethylene glycol) dimethacrylate and two methacryloxymodified silicas (Aerosil R7200 and R711) were investigated to find possible correlation between the stability of the monomer/silica dispersion, curing kinetics, composite morphology and physical and mechanical properties of the final hybrid material. The monomer/silica dispersions were cured photochemically. The investigated parameters: Zeta potential, polymerization rate and conversion, glass transition, surface roughness and mechanical properties were found to be synchronous; when plotted as function of silica content, they showed maxima or changes in the trend at the same filler loading. This threshold (optimum) silica content in the composition was about 5 wt. $-\%$ for the investigated systems. The results obtained are discussed in terms of the solvation cell (which influences dispersion stability) and the interphase layer formation as well as their changes below and above of the threshold filler content.
\end{abstract}

Keywords Nanocomposites $\cdot$ Silica $\cdot$ Photopolymerization · Zeta potential $\cdot$ Curing kinetics $\cdot$ Surface morphology

Ewa Andrzejewska

ewa.andrzejewska@put.poznan.pl

1 Faculty of Chemical Technology, Poznan University of Technology, Berdychowo 4, 60-965 Poznan, Poland

2 Faculty of Technical Physics, Poznan University of Technology, Piotrowo 3, 60-965 Poznan, Poland

3 Department of Theory \& Bio-Systems, Max Planck Institute of Colloids and Interfaces, Am Mühlenberg 1 Golm, 14476 Potsdam, Germany

\section{Introduction}

In recent years hybrid inorganic-organic nanocomposites were deeply investigated for their exciting bulk and surface properties. These materials combine the high ductility and low-temperature processing conditions of polymers with the outstanding properties of ceramics, such as hardness, high strength and modulus, thermal stability as well as low coefficient of thermal expansion [1]. The inorganic component is usually constituted of either layered clays, or nanoparticles with wide variety of different shapes (discs, rods, spheres). The properties of the resulting polymer nanocomposite depend on the characteristics, dimensions, and shapes of the inorganic fillers and also on the interfacial bonding strength. However, it is very difficult to manufacture nanocomposites with the nanoparticles dispersed uniformly because nanoparticles have very high surface energies and are prone to agglomerate into larger particles during the preparations of materials. In unmodified silica for example, the presence of hydroxyl groups can lead to hydrogen bonding between contiguous particles. Therefore, the surface modification of nanoscale fillers becomes necessary in preparation of nanocomposites.

Polymer nanocomposites exhibit unusual combinations of properties and unique design possibilities. Addition of silica to polymer can improve the physical properties such as the mechanical properties and thermal properties of the materials. Of particular interest is the methacrylate-silica hybrid, which has found great use in coatings, optical devices and membrane application. While methacrylics provide easy processability and optical transparency, nanosilica particles provide mechanical properties (e. g. hardness, scratch resistance). The scale of phase separation in hybrid systems is thought to be most essential to the formation of transparent composite. It is important to recognize that homogeneity and phase behavior of 
hybrid materials is related to the organic-inorganic interfacial interaction. With this strong bond interaction at the organicinorganic interface, hybrid material forms homogeneous phase and becomes transparent, where chemical or hydrogen bonding binds the organic phase to the inorganic matrix and prevents occurrence of macrophase separation [2].

Organic-inorganic polymer nanocomposites can be rapidly produced at ambient temperature by photocuring of systems containing monomers and dispersed nanoparticles. Besides its environment-friendly aspect (no emission of volatile organic compounds, low energy consumption), photopolymerization technology offers a number of advantages, namely, ultrafast curing, ambient temperature operation, spatial and temporal control of the process, and wide range of mechanical properties of the highly crosslinked polymer formed [3].

As a subdivision of hybrids, UV-curable hybrid materials show the synergistic unique properties to these materials, finally finding potential uses in fields such as printing, inks, adhesives, device packaging and especially clear coats $[1,4,5]$. Recently, photocurable hybrids based on silica have been applied in medicine to create polymer networks for use in applications such as bone and dental restorations [6], coatings [7], for artificial implants and functional hydrogel materials [8].

Photopolymerization kinetics of (meth)acrylate monomers containing dispersed nanosilica were considered in several papers and various dependences of the polymerization rate and conversion on silica content were obtained; the results were explained in various ways [9-12]. Ours previous papers $[8,13,14]$ analyzed kinetics more deeply (in the considered cases a maximum on the dependence of the polymerization rate on the silica content was observed). On the other hand, mechanical properties of this type of composites were often investigated (e.g. [15-19]) and various effects of silica addition were observed. These effects were attempted to correlate with silica content, surface modification, particle size, interphase formation, etc. However, when a composite is formed in situ by curing of a monomer/silica mixture, an important issue becomes stability of the filler dispersion which can determine not only the morphology of the resulting composite but also may affect curing kinetics. Until now there were no reports with a broader view on this subject, which would try to correlate all these issues: stability of the dispersion (understood as its susceptibility to flocculation), curing kinetics, composite morphology and physical and mechanical properties of the cured material. This attempt has been undertaken in the present work. The model systems were based on polyethylene glycol dimethacrylate (PEGDMA, matrix precursor) and two commercial modified silicas: Aerosil R7200 and Aerosil R711. Multifunctionality of the monomer ensured formation of a cross-linked matrix. The both Aerosils are fumed silicas with primary particle size $12 \mathrm{~nm}$, after treated with methacrylsilane; Aerosil R7200 has been additionally structure modified to reduce the size of agglomerates [20].
Results presented in this work show that parameters which characterize the above discussed properties of a formulation before, during and after curing: Zeta potential, polymerization rate and conversion, glass transition, surface roughness and mechanical properties are synchronous; when plotted as function of silica content, they show maxima or changes in trend at the same filler loading.

\section{Experimental}

\section{Materials}

The monomer, polyethylene glycol dimethacrylate with $\mathrm{MW}=550$ (PEGDMA, $n=9$ ) was purchased from Aldrich and was purified by column chromatography before use (Scheme 1). The photoinitiator, 2,2-dimethoxy-2phenylacetophenone (Irgacure 651) was supplied by SigmaAldrich. Aerosil R7200 and Aerosil R711 were kindly donated by Evonik. According to the supplier they have the same specific area $\left(125-175 \mathrm{~m}^{2} / \mathrm{g}\right)$ [20]. The silicas were dried at $110{ }^{\circ} \mathrm{C}$ for $2 \mathrm{~h}$ before use. The monomer/filler mixtures were homogenized by ultasonication by $10 \mathrm{~h}$. All the formulations investigated in this work were transparent and did not show any visual evidence of precipitation over long periods of time.<smiles>C=C(C)C(=O)OC(C)(C)COC(C)(C)C(=O)C(=C)C</smiles>

Scheme 1 Chemical structure of PEGDMA

\section{Methods}

\section{Viscosity}

Viscosities of monomer/silica mixtures were measured at the polymerization temperature $\left(40^{\circ} \mathrm{C}, \eta^{40}\right)$ at $0-160 \mathrm{rpm}$ with a Digital Viscometer (model DV-II, Brookfield, USA) in coneplate geometry.

\section{Dynamic light scattering}

Dispersions of the silicas in the monomer were characterized with a ZetasizerNano ZS (Malvern Instruments Ltd., United Kingdom). Particle size and particle size distribution (PSD) were measured employing the technique of non-invasive back scattering method (NIBS) with a constant $173^{\circ}$ scattering angle, at $(25 \pm 0.1)^{\circ} \mathrm{C}$. 


\section{Photopolymerization kinetics}

Reaction rates $\left(R_{p}\right)$ and conversions $(p)$ were determined by DSC under isothermal conditions at $40 \pm 0.01{ }^{\circ} \mathrm{C}$ in a highpurity argon atmosphere $\left(<0.0005 \%\right.$ of $\left.\mathrm{O}_{2}\right)$ using the Pyris 6 instrument (Perkin-Elmer, USA) equipped with a lid specially designed for photochemical measurements. The 2-mg samples were polymerized in open aluminum pans with the diameter of $6.6 \mathrm{~mm}$. The polymerizations were initiated by the light from a LED Hamamatsu LC-L1 lamp $(\lambda=365 \mathrm{~nm}$, light intensity at the sample pan position $2.75 \mathrm{~mW} \cdot \mathrm{cm}^{-2}$, photoinitiator concentration 0.2 wt.-\%). All DSC photopolymerization experiments were conducted at least in triplicate. The reproducibility of the results was about $\pm 3 \%$.

\section{Glass transition temperature $T_{g}$}

Glass temperature $T_{g}$ of the composites was measured by DSC technique using Pyris 6, Perkin Elmer instrument. The $2 \mathrm{mg}$ samples were scanned at a heating rate of $10{ }^{\circ} \mathrm{C} \mathrm{min}{ }^{-1}$ in temperature range from -40 to $120^{\circ} \mathrm{C}$.

\section{AFM measurements}

Specimens for AFM imaging were prepared by photopolymerization in DSC sample pans in the DSC instrument under Ar atmosphere, thereafter cut vertically along the diameter and measured on the upper side close to the center of the cutting edge in the tapping mode. This AFM mode represents very good vertical resolution together with its nondamageable work at high set-points (an AFM tip only gently contacts the surface once at the oscillation period) that might physically affect or modify the compliant surfaces. The tapping mode is also effective to analyze a phase composition of surfaces and their behavior at interfaces, which is excellent for our purpose.

Height and phase images were obtained in the Dimension 3100 AFM controlled by the Nanoscope IIIa controller (Bruker/Digital Instruments, USA). Standard tetrahedral silicone cantilevers (model OMCL-AC160TS, Olympus, Japan) of spring constant $\sim 26 \mathrm{~N} \mathrm{~m}^{-1}$, resonance frequency $\sim 300 \mathrm{kHz}$, and tip radius $\sim 7 \mathrm{~nm}$, were used. These cantilevers are characterized by a steep phase shift at the vicinity of the resonance frequency, and therefore they provide notable phase contrast for sample surface composition. Occasionally, randomly chosen samples were scanned using super sharp silicon tips (model Hi'Res-C15/Cr-Au, $\mu$ Mash, USA) of radius $\sim 1 \mathrm{~nm}$ (spring constant $\sim 40 \mathrm{~N} \mathrm{~m}^{-1}$, resonance frequency $\sim 325 \mathrm{kHz}$ ) to compare the obtained morphologies. The samples were measured in air at room temperature at stable value of relative humidity $(40 \%)$ in a low-noise acoustic chamber. For the evaluation of the AFM data Gwyddion 2.38 and NanoScope Analysis 1.50 were used.
For the height images the mean roughness $R a$ was calculated for the scan area of $5 \times 5 \mu \mathrm{m}^{2}$ as this area is an optimum scan range that, on one hand, is small enough to be adapted to nanoparticle structures (the number of scan points per line is fixed and this means that the smaller the scan area the better the resolution), on the other hand, large enough to minimize possible influence from individual non-homogenous features. The mean roughness $R a$ is defined as the average deviation of the profile from a mean line. $R a$ is commonly used as a "onevalue" parameter that describes the surface profile in homogenous samples; it provides much reliability in statistical surface measures. Roughness measures were done at 2 different spots on each specimen (samples were triplet, i.e. prepared under the same conditions).

\section{Mechanical testing}

For mechanical testing the samples were cured in a steel mold with polyethylene terephthalate (PET) cover and the whole spectrum of a DYMAX-Blue Wave 50 has been applied. The mechanical properties were investigated according to standards PN-ISO 868:2004 and PN-EN ISO 527-1:1998 with the use of a universal testing machine (Z020 Zwick Roell) and a Shore hardness testing machine (Zwick Roell).

\section{Results and discussion}

Investigations were performed for a series of compositions containing 2, 4, 5, 10 and $15 \mathrm{wt} .-\%$ of silica. The compositions were characterized before the polymerization (particle size distribution, viscosity, Zeta potential), during the polymerization (polymerization rate and conversion as functions of time) and after curing (glass transition, surface morphology and roughness, tensile properties, hardness).

\section{Particle size distribution}

Particle size distributions PSD (Table 1) in formulations show the existence of aggregates/agglomerates in the submicro range (about 100-1000 $\mathrm{nm}$ ). The size of particle clusters increases with the silica content. Only formulations containing highest amounts of silica show a bimodal distribution of agglomerates (which is reflected by higher values of polydispersity index PDI). The size of aggregates/agglomerates of R711 in the monomer is larger than that of R7200. These PSD results could suggest that after the polymerization the obtained materials would be microcomposites; however, AFM results indicate that the particle clusters on the composite surface are of the nano size (as will be discussed later). This discrepancy may result from the fact that DLS method provides an intensity and volume diameter which are particularly sensitive to the presence of larger particles and in this way overrates the 
Table 1 Mean particle size diameter and polydispspersity index PDI of silica particles dispersed in the monomer

\begin{tabular}{|c|c|c|c|c|c|c|}
\hline \multirow{2}{*}{$\begin{array}{l}\text { Silica } \\
\text { (wt.-\%) }\end{array}$} & \multicolumn{3}{|l|}{ Aerosil R7200 } & \multicolumn{3}{|l|}{ Aerosil R711 } \\
\hline & $\begin{array}{l}\text { Range of particle } \\
\text { diameters (nm) }\end{array}$ & $\begin{array}{l}\text { z-average } \\
\text { diameter (nm) }\end{array}$ & $\begin{array}{l}\text { Polydispspersity } \\
\text { index PDI }\end{array}$ & $\begin{array}{l}\text { Range of particle } \\
\text { diameters (nm) }\end{array}$ & $\mathrm{z}$-average diameter $(\mathrm{nm})$ & $\begin{array}{l}\text { Polydispspersity } \\
\text { index PDI }\end{array}$ \\
\hline 2 & $106-295$ & 259 & 0.100 & $142-295$ & 235 & 0.409 \\
\hline 4 & $142-295$ & 247 & 0.446 & $342-825$ & 281 & 0.231 \\
\hline 5 & $255-531$ & 270 & 0.396 & $295-712$ & 413 & 0.119 \\
\hline 10 & $396-955$ & 467 & 0.498 & $220-396 ; 955-1100$ & $301 ; 1032$ & 0.665 \\
\hline 15 & $190-342 ; 712-1480$ & $266 ; 1060$ & 0.670 & $190-342 ; 1280-1720$ & $266 ; 1493$ & 1 \\
\hline
\end{tabular}

actual size [21]. This method measures the hydrodynamic diameter of aggregates. The hydrodynamic diameter is that of a sphere that has the same translational diffusion coefficient as the aggregate being measured. Thus, it is expected that in the monomer/silica mixtures the size of aggregates which are not perfectly spherical, is always larger than in the reality. This can explain in part transparency of dispersions despite seemingly large aggregates/agglomerates.

Another important factor is that the transparency of dispersion depends also on the difference between refractive indices of the monomer (PEGDMA) and the filler material (Aerosil). Aerosil products ( $n=1.46$ ) [20] have practically no refractive index contrast with PEGDMA $(n=1.460)$, therefore the materials can be transparent.

\section{Viscosity}

The importance of viscosity as a parameter characterizing the formulation is associated with its influence both on curing kinetics as well as on the processability of the system [22, 23]. It usually reflects particle-particle and particle-monomer interactions. Such interactions are possible due to the presence of silanol groups on the silica surface. Although the silicas used in this work were surface modified with methacryloxy groups, they still contain a number of silanol functions giving a possibility to interact (which was confirmed by the presence of absorption bands from $\mathrm{SiOH}$ groups in the FTIR spectrum - data not shown - and the producer information [20]). Figure 1 shows the viscosity of the monomer/silica dispersions as a function of the shear rate and the content and type of the filler. The measurements were performed at $40{ }^{\circ} \mathrm{C}$, which is the temperature of kinetic studies and composite preparation. As could be expected, viscosity of the composition increases with the silica content. The viscosity of the neat monomer exhibits Newtonian behavior; such a behavior is observed also in the case of silica R7200-containing formulations (up to about $10 \%$ of silica loading) indicating the lack of network formation by silica particles as a consequence of surface modification (strong interactions between silica particles lead usually to non-Newtonian behavior). Interactions between the monomer and the filler prevent the formation of silica network; therefore the Newtonian behavior of viscosity can indirectly indicate the existence of silica/monomer interactions. Such interactions in turn lead to formation of a solvation layer which separates filler particles from each other.

Formulations containing silica R711 have markedly higher viscosities and show a slight shear thinning behavior. This in turn could suggest stronger interactions between silica R711 particles. The exact surface nature of the both types of silica, e.g. degree of surface modification, is not known; therefore it is difficult to indicate the strict reason of such a behavior.

\section{System stability}

Although there are many reports on the effect of silica addition on various properties of monomer/silica formulation and the resulting composite, there are no reports on the stability of the formulation understood as its susceptibility to flocculation (this issue was only briefly mentioned in our previous works $[13,14])$.

Stability of dispersion can be achieved by two main mechanisms, i.e. electrostatic stabilization (repulsive interactions) and steric stabilization (which consists in covering the particles with polymer preventing them to get closer in the range of attractive forces). Electrostatic stabilization is mainly considered for silica suspensions in water. In non-aqueous media it

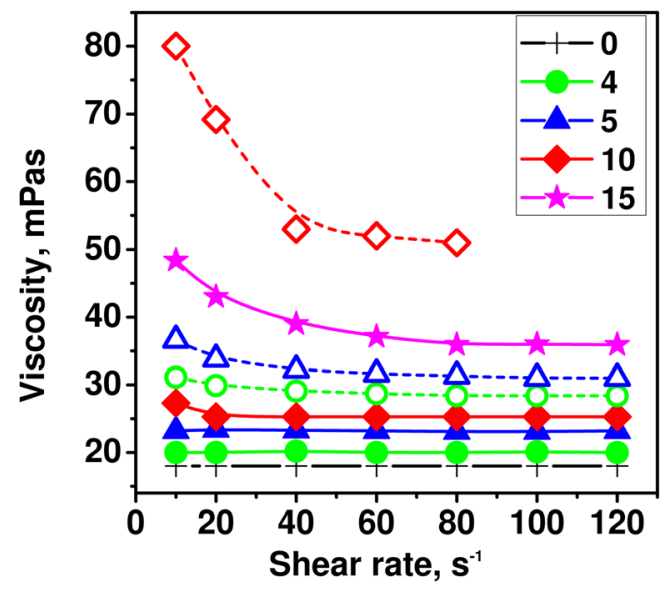

Fig. 1 Viscosity of formulation as a function of the shear rate and silica loading at $40{ }^{\circ} \mathrm{C}$. Numbers indicate silica content in wt.- $\%$. Filled symbols: R7200-containing formulations, open symbols: R711containing formulations. The lines are guides to the eye 
can readily occur in highly polar solvents with high dielectric constant $(\varepsilon>11)$ but is also possible in semipolar liquids $(5<\varepsilon<11)[24,25]$ or even in solvents of very low $\varepsilon$, like 2 [26]. End-capped polyethylene glycols have $\varepsilon$ of the order of 6-10 [24], thus, electrostatic stabilization in PEGDMA should be possible. The charging mechanism is based on electrondonor interactions between the dispersing agent and the particle surface, i.e. formation of an electron-donor acceptor complex at the surface (not bare silica-liquid interaction [27]). In our case the interaction would occur between $\mathrm{Si}-\mathrm{OH}$ group on the silica surface and the monomer ether and carbonyl groups.

Electrostatic stabilization can be determined by the Zeta potential. It describes characteristic, repulsive interactions of particles with the same charge in dispersion. In the case of silica particles, silanol groups present at their surface yield a negative charge and the organic donor molecules (monomer) yield a positive charge forming the electrical double layer. The electrostatic repulsion arises when the electrical double layers around the filler particles begin to interpenetrate. Due to the relation between the Zeta potential and repulsive forces between particles, a higher charge (and higher absolute value of Zeta potential) will give a more stable colloidal system.

Zeta potential of the investigated formulations as a function of the silica content is shown in Fig. 2.

As stated above, the surface of silica particles is negatively charged (negative Zeta potentials) indicating on the dominant acceptor properties of the silica surface towards the monomer molecule. Zeta potential at first decreases with the silica loading and reaches a minimum value (highest absolute value) at about $4-5$ wt.- $\%$ of silica content; thus, the formulations containing about 4 wt.- $\%$ of silica seem to be best stabilized electrostatically. Further addition of silica increases Zeta potential (decreases its negativity) indicating on stepwise reduction of dispersion stability. It can be associated with the fact that electrostatic stabilization at high particle concentration is not possible in non-aqueous systems [28]. Stability of dispersions containing silica R7200 seems to be somewhat better that those containing R711, which can be associated with smaller

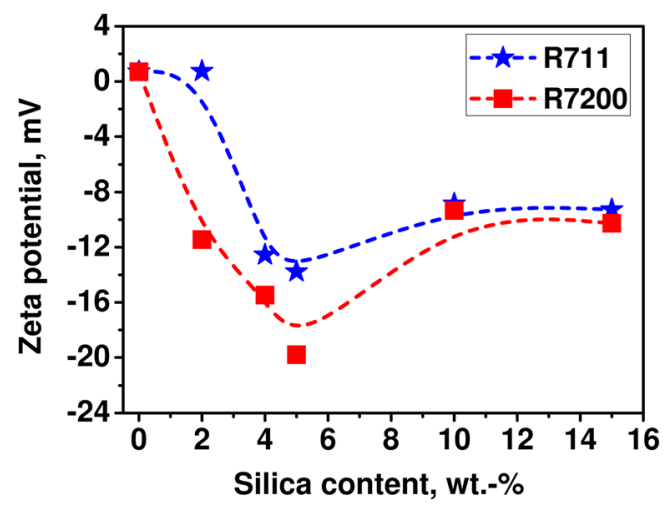

Fig. 2 Zeta potential values as a function of Aerosil 7200 and Aerosil 711 content in PEGDMA at $40{ }^{\circ} \mathrm{C}$. The lines are guides to the eye agglomerates of the former filler. The existence of the electrostatic stabilization suggests the presence of a solvation cell around the filler particles, which during the polymerization will be converted to an interphase region in which polymer chains interact with silica particles. This in turn will shape composite properties.

\section{Photopolymerization kinetics}

Stability of the dispersion and its viscosity can have an impact on the curing kinetics. The conversion-rate curves for the photopolymerization of formulations containing various amounts of silica R720 are shown in Fig. 3a.

PEGDMA is a multifunctional monomer (containing two double bonds) and the shape of the kinetic curves is
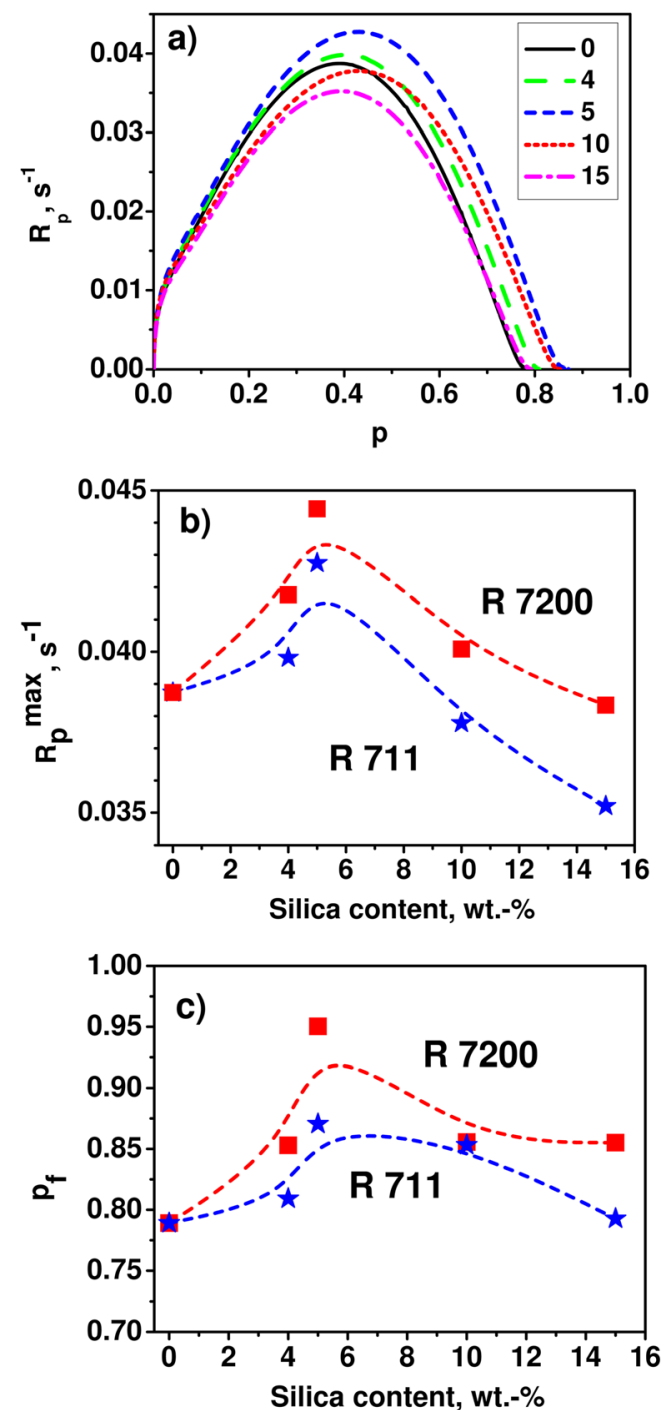

Fig. 3 The dependence of the polymerization rate $R_{p}$ on double bond conversion $p$ at $40{ }^{\circ} \mathrm{C}$ for the monomer/Aerosil R711 system at various silica content (in wt.-\%) (a), maximum polymerization rate $R_{p}{ }^{\max }(\mathbf{b})$ and final conversion $p^{f}$ as functions of Aerosil R7200 and Aerosil R711 content (c). The lines are guides to the eye 
characteristic for a crosslinking polymerization with immediate onset of autoacceleration, occurrence of the maximum polymerization rate $R_{p}{ }^{\text {max }}$ and incomplete conversion of double bonds. Addition of silica influences the polymerization kinetics causing changes in the polymerization rate $R_{p}$ and final conversion $p^{f}$. The maximum polymerization rate $R_{p}{ }^{\max }$ appears in the range of $35-43 \%$ of double bond conversion. It corresponds to the reaction point at which reaction diffusion becomes the dominating mechanism of termination [3] and is usually associated with inability of macroradicals to translational diffusion. This parameter at first increases with the silica content and then reaches the highest value for composition containing 5 wt. $-\%$ of the silica; further increase in silica loadings reduces the maximum polymerization rate. Too high filler content (above about $10-15 \mathrm{wt} .-\mathrm{\%}$ in the case considered) can even retard the polymerization. An analogous behavior show also formulations containing Aerosil R7200 but the polymerization rates in this case are higher (Fig. 3b). The same dependence on silica loading occurs in the case of final conversion (Fig. 3c). It is worth to note that conversion of double bonds depends on system mobility: an increase in chain mobility usually leads to enhanced conversion. Because silica particles hinder movements of the polymer chains, we should expect rather a decrease in conversion. Thus, the increase in conversion in the presence of silica is probably associated with the beneficial influence of silica on the polymerization rate (deceleration begins at higher conversion, Fig. 3a). This type of the accelerating behavior of silica has been also reported earlier $[8,13,29]$ and many possible explanation were proposed but only our earlier reports took into account a possible influence of dispersion stability on curing kinetics [13, 30]. Inspection of Figs. 2 and $3 \mathrm{~b}$ allows finding the relationship: the maximum polymerization rate increases as the stability of the formulation (measured by the absolute value of Zeta potential) increases; after reaching their maxima the both parameters begin to decrease.

Accelerating effect of silica addition on the polymerization of another dimethacrylate was explained by us in ref. [13] by an increase in the propagation rate coefficient $k_{p}$ (no reason was proposed) and a slight decrease in the termination rate coefficient $k_{t}$ (associated with the increase in viscosity of the formulation); moreover, slightly higher $k_{p}$ values showed compositions containing Aerosil R7200 than those with Aerosil R711. The increase in the polymerization rate does not result simply from the enhanced total concentration of the polymerizing groups after addition of methacryloxygrafted silica, because a similar effect is observed also in the presence of non-modified silicas [8]. Our considerations concerning the influence of dispersion stability prove that the polymerization is faster in case of formulations showing higher absolute Zeta potential values, both resulting from the silica content as well as silica type. Thus, we may suspect that the increase in $k_{p}$ can be associated in a way with the stability of the dispersion. It is possible that the formation of a solvation cell can increase the local concentration of double bonds leading to an apparent increase in $k_{p}$ and the interphase which begins to form during the polymerization immobilizes macroradicals leading to suppression of termination.

If the stability of the dispersion influences the solvation cell and thus the interphase layer, this should be reflected in the morphology of the composite.

\section{Glass transition}

One of the most important parameters characterizing polymeric materials is the glass transition $\left(T_{g}\right)$. The deviation in $T_{g}$ of composites from the neat polymer is controlled by two primary factors: the dispersion state of the nanofiller and the modified mobility of the polymer in the interfacial region [31].

Poly-PEGDMA is an elastomer with the glass temperature about $-10{ }^{\circ} \mathrm{C}$ (Fig. 4). Addition of silica increases $T_{g}$ by several degrees in the case of R711 and only slightly in the case of R7200. Generally, the increase of the glass transition is due to the topological constrains wagered by rigid silica domains to movement of polymer chain segments. The slight increase of $T_{g}$ may indicate that polymer/silica interactions are not strong and the interphase is rather thin. Somewhat higher $T_{g}$ values in the case of silica R711-containing composites may be due to the fact that larger filler particles produce a greater increase in $T_{g}[8]$.

The increase of $T_{g}$ is not monotonic and this parameter reaches a maximum at the silica content about 5 wt.- $\%$. It is interesting that this composition corresponds to the most stable formulation before curing indicating on a relationship between the two parameters. At lower silica content $T_{g}$ increases due to reduction of the segmental mobility of the chains near the filler particles and as a result of attractive interactions with the polymer matrix in the interfacial region. The amount of interfacial area between the polymer and silica increases with filler content causing the increases in $T_{g}$. However, when $T_{g}$ begins to decrease with increasing silica content (above 5 wt.-\%), it may indicate on a partial loss of the compatibility

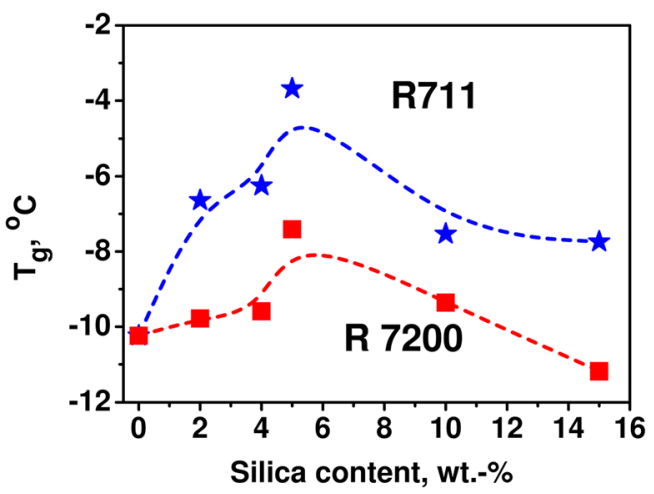

Fig. 4 Glass transition temperature $T_{g}$ as a function of silica (R7200 and $\mathrm{R} 711$ ) content. The lines are guides to the eye 
with the polymer matrix (caused by too high concentration of the filler). This in turn may be associated with severe agglomeration of particles [32] and is in agreement with the results of Zeta potential measurements showing increasing susceptibility of dispersions to flocculation. Thus, as long as the electrostatic stabilization increases with the increase of silica loading, probably the amount of the interphase in the composite also increases leading to the increase in $T_{g}$ until it reaches maximum. Further increase in silica loading leads to such increase in the size of agglomerates that electrostatic stabilization becomes less and less efficient; the total amount of solvation layer and the deriving interphase in the composite decrease resulting in the stepwise decrease of $T_{g}$. Only in the case of silica R711-containing composites the reinforcing effect dominates in the whole range of the filler content ( $T_{g}$ values are higher than that of the neat poly-PEGDMA).

\section{AFM}

Topographical analysis of silica aggregates and morphological analysis of their dispersion in the polymer was carried out using AFM.

Figures 5 and 6 present AFM height and phase images, respectively, performed for the neat polymer matrix and composites containing 4,10 , and 15 wt.- $\%$ of silica R7200 and
R711. The images clearly indicate changes occurring in the composite surface structure with increasing silica loading.

The neat poly-PEGDMA sample (Fig. 5a) is smooth (see section analysis plot) and homogenous with no apparent surface features like cracks, voids and peaks, with the mean roughness of $6.3 \mathrm{~nm}$ (Fig. 7). This confirms also the phase image (Fig. 6); a uniform color in the AFM image manifests the same sort of mechanical compliance of the sample for the neat polymer. In general for all composites, the estimated size of aggregates is about $30 \mathrm{~nm}$ to $90 \mathrm{~nm}$ in diameter, i.e. only a few single nanoparticles in an aggregate, thus, the obtained material can be regarded as a nanocomposite. This result differs from that obtained by determination of the particle size in solution (by DLS), as was pointed out earlier. Phase image (Fig. 6) shows a significant contrast of silica and polymer part. Bright color reveals distinct features of polymer-covered silica particles that are surrounded with the matrix (dark color). Small addition of the silica nanoparticles to the polymer matrix influences greatly the surface structure of the final composites, e.g. only 4 wt.- $\%$ of the filler leads to significant changes in the sample topography and morphology (Figs. 5b and e, 6 and 7), however, in a different way for silica R7200 and R711. In the case of silica R7200 (4 wt.-\% of the filler) the particles are discretely distributed in the polymer matrix; they do not form continues connection as the polymer phase fraction dominates between them (Fig. 5b). In the case of silica

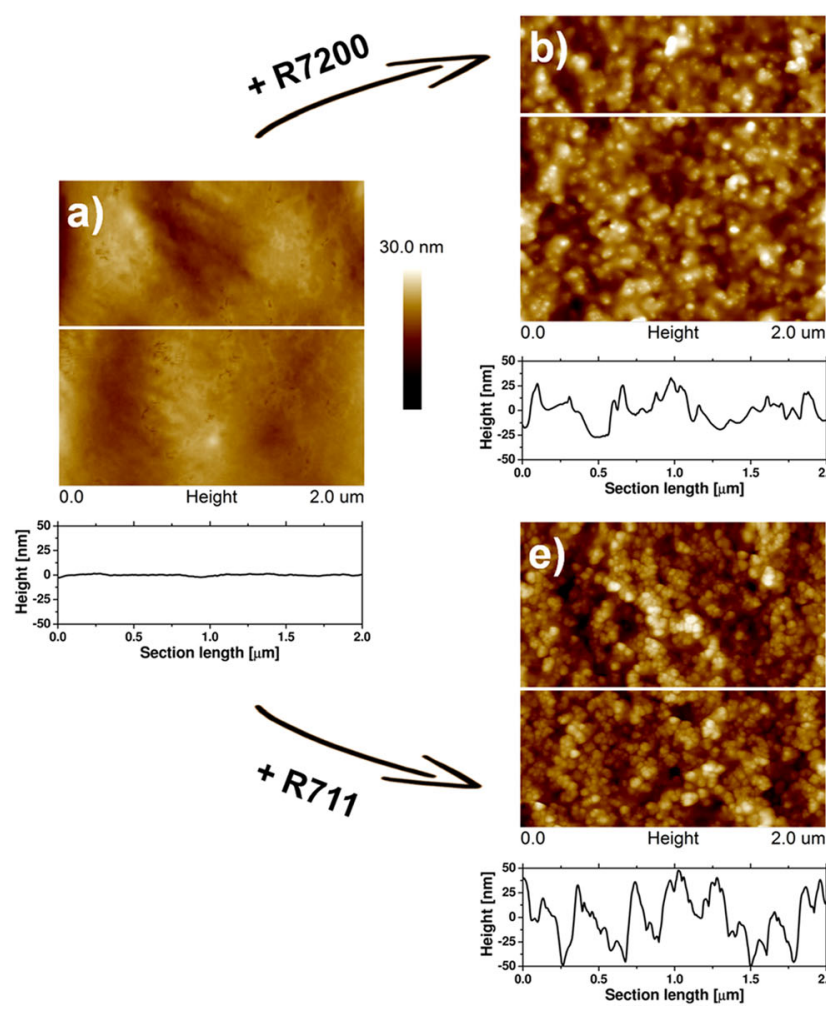

Fig. 5 AFM height images of (a) neatPEGDMA, and polyPEGDMA filled with (b) 4 wt.- $\%$, (c) 10 wt.- $\%$, (d) 15 wt.- $\%$ of silica R7200, and (e) 4 wt.- $\%$, (f) 10 wt.- $\%$, (g) 15 wt.- $\%$ of silica R711. The height-profiles
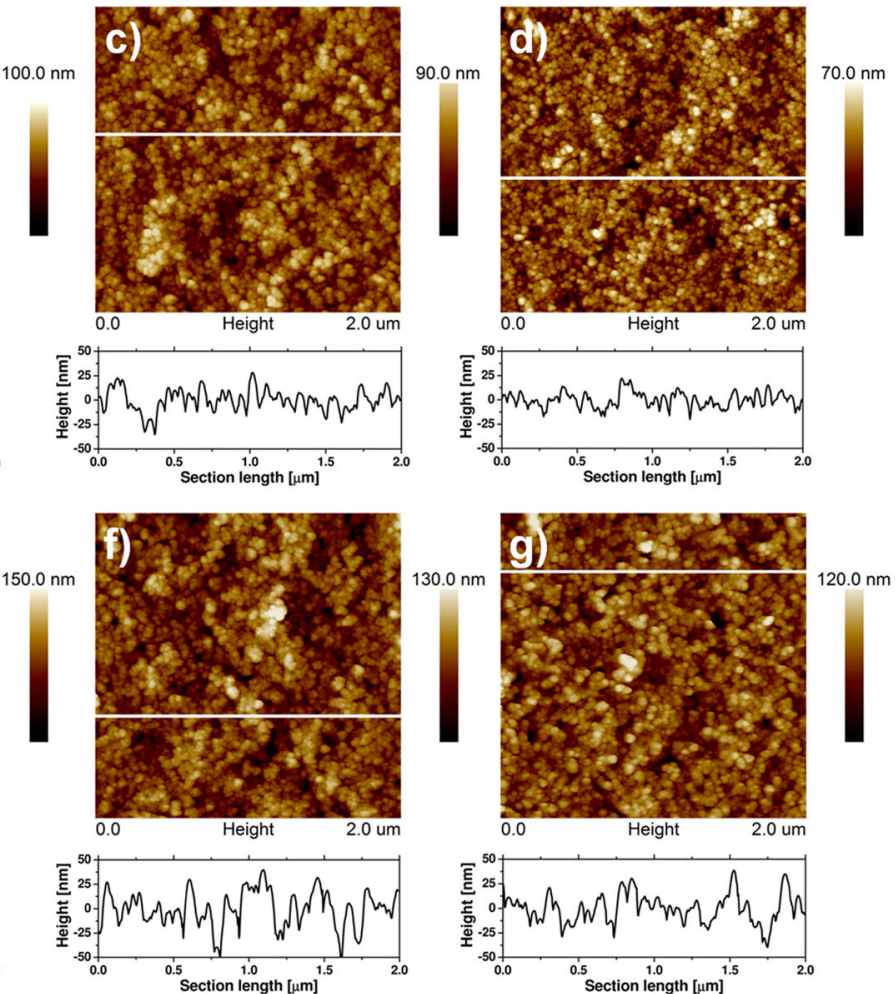

(cross-section) were taken along the white lines; for all the height-profiles the height axis was adjust to $100 \mathrm{~nm}$ to get the profiles comparable 


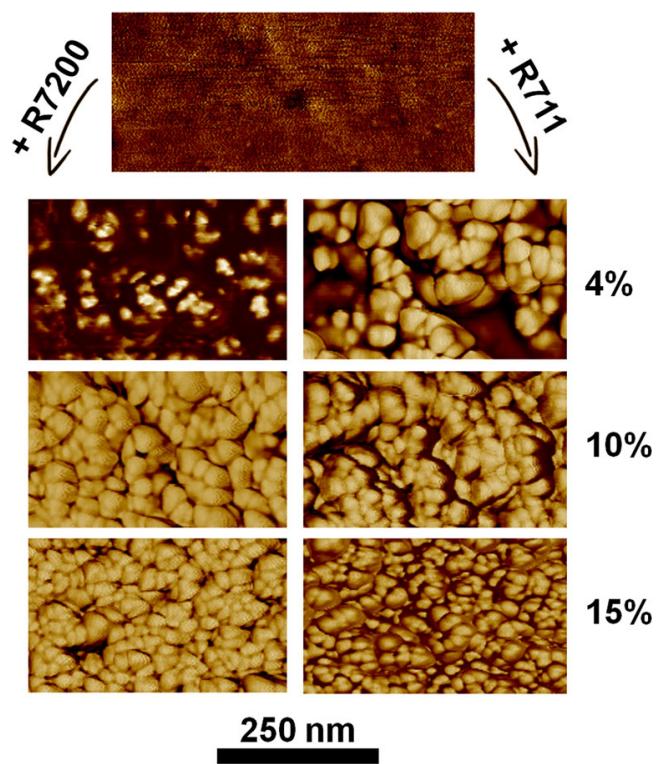

Fig. 6 High-resolution AFM phase images of neatPEGDMA surface, and its respective composites based on silica R7200 and R711 (as indicated). The color scale is encoded from dark (PEGDMA phase) to bright (PEGDMA + silicas phase)

R711 (4 wt.- $\%$ of the filler) the particles form continues connection of aggregates with a small polymer phase fraction between them (Figs. 5e and 6). This indicates on stronger tendency of this silica to be localized at the interface. Such a behavior can be associated in part with lower availability of methacryloxy groups on the surface of silica particles clustered in larger aggregates. In such a case the ratio of the rates of two competing processes which take place during the curing: chemical incorporation into the polymer matrix being formed and diffusion to the surface is less favorable.

Once the silica concentration in the polymer is larger than 10 wt.- $\%$, all the composites show continuous phase of nanoparticles and their aggregates, surrounded by a thin layer of matrix (Figs. 5c, d, f, g, and 6). The aggregates are densely dispersed at the surface, however, their uniformity is notably improved for higher fillings, e.g. $15 \mathrm{wt.}-\%$. This could be seen directly in high resolution phase images in Fig. 6 and section

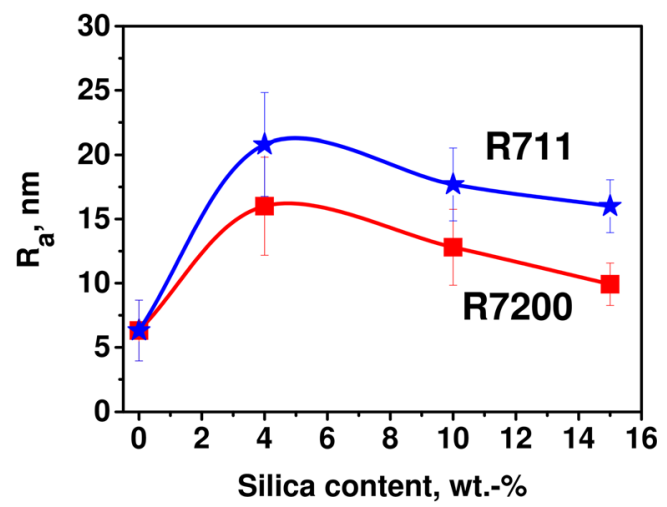

Fig. 7 Mean roughness $R a$ as function of silica content. The lines are guides to the eye profiles in Fig. 5, where the amplitude of the section profile, i.e. the maximum height, is reduced; it is especially visible for the composite containing $15 \mathrm{wt} .-\%$ of silica R7200.

Additional (quantitative) analysis of the surface structure of the investigated composites was done by mean roughness, $R a$, measurements. The $R a$ initially strongly increases with the silica loading, approx. 3-4 times at the maximum reached at 4 wt.-\% (16.0 nm and $20.8 \mathrm{~nm}$ for R7200 and R711-based composites, respectively), and then decreases gradually. This result indicates that when the silica content exceeds $\sim 4$ wt.- $\%$ the uniformity of its dispersion increases. Higher $R a$ values in the case of the Aerosil R711-based composites is in agreement with larger particles of this silica.

The appearance of the maximum value of the mean roughness at about 4 wt.- $\%$ of silica loading corresponds with the results obtained from dispersion stability, kinetic and $T_{g}$ investigations. As discussed earlier, above the threshold filler content the amount of the interphase decreases which along with larger number of aggregates/agglomerates leads to more uniform surface and smaller roughness.

\section{Mechanical properties}

Determination of mechanical properties of the investigated composites included Young's modulus $(E)$, tensile strength $\left(\sigma_{M}\right)$, elongation to break $\left(\varepsilon_{M}\right)$ and hardness (Fig. 8).

The Young's modulus increases almost linearly with the increase of the filler content and is practically the same for two types of composites (with R7200 and R711). The increase is significant: by about $46 \%$ at 5 wt.- $\%$ and by about $130 \%$ with 15 wt.- $\%$ for each silica. In general, the overall nanocomposite stiffness is influenced by the stiffness of the matrix and the filler, the filler dispersion, and the stiffness and connectivity of the interphase [33]. In our case the stiffness of the filler seems to dominate; as reported, the increase in stiffness of nanocomposites is a result of the high modulus of the particulate fillers (modulus of silica $=70 \mathrm{GPa}$ ) [19].

The tensile strength also increases markedly with silica loading (Fig. 8 b), by about $43 \%$ at 5 wt. $\%$ and even by about $85-100 \%$ at 15 wt. $\%$ of the filler content. The increase in tensile strength proves that the mechanical loading is effectively transferred from the matrix to the filler particles owing to the interfacial bonding effect due to surface modification. However, we can observe a change in the slope of this dependence at about 5 wt.- $\%$ of silica, well-marked for R711 and very slight for R7200. Slowdown in the increase of the tensile strength above $5 \mathrm{wt} .-\%$ of silica content can reflect a change in the dispersion status of the fillers, which is in agreement with the results presented above. Poorer filler dispersion and reduced filler-matrix interaction due to decreased amount of the interphase, especially for composites with R711, leads the observed effect. The ductility of the composites against the filler content is shown in Fig. 8c. Up to about 5 wt.-\% of 

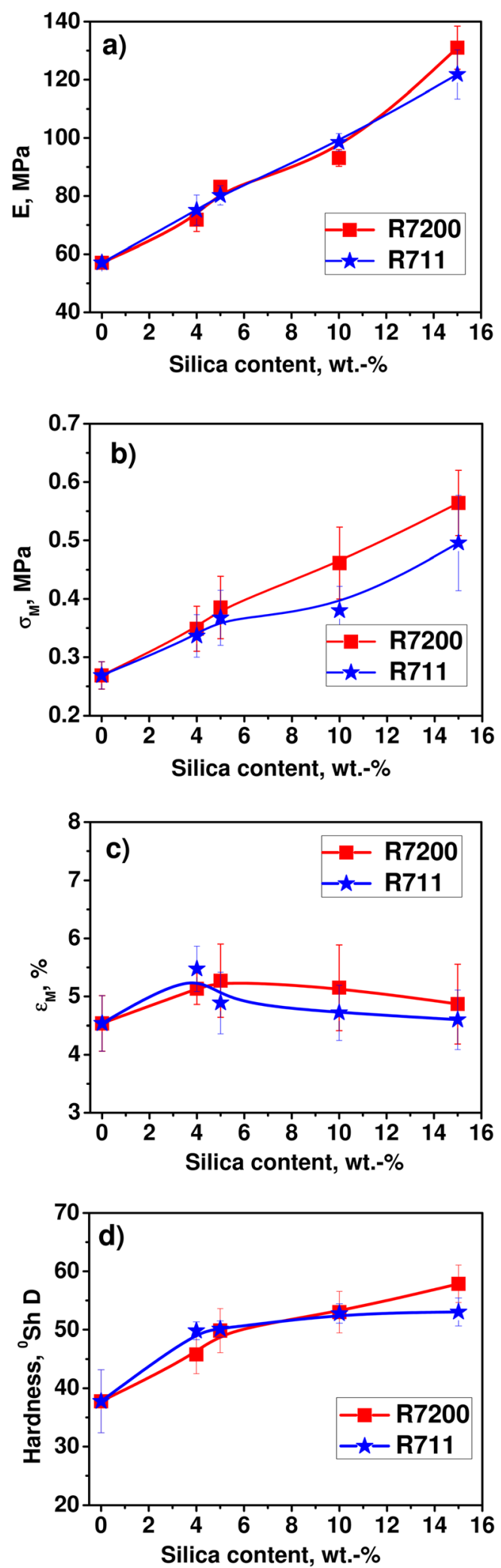

Fig. 8 Young modulus (a), tensile strength (b), elongation at break (c) and hardness (d) of the composites containing various amounts of silica R7200 and R711. The lines are guides to the eye

silica loading elongation to break increases slightly (by $16 \%$ ). This indicates on the toughening effect of the particles on the polymer matrix, which can be associated with the increased contribution of the interfacial viscoelastic deformation. Increase in ductility proves the existence of a flexible interface layer. The tendency of decrease in elongation to break above the filler content of about $5 \mathrm{wt} .-\%$ suggests that matrix deformation is not only related to the interface feature but also to the dispersion state of the fillers. The slight decrease in ductility indicates a reduction in the matrix deformation due to the introduction of mechanical restraints by the almost nondeformable filler particles. Different elastic properties of the material components cause that the particles act as stress concentrators. Particle agglomeration promotes higher stress concentration, a more extensive cavitation and therefore a faster breaking [34]. Thus, the appearance of the maximum on the $\varepsilon_{M}=f($ silica content $)$ dependence is again in agreement with our other results.

Despite the very similar ductility behavior of the two types of composites, there is a small difference in values of elongation to break. The higher ductility values of composites containing silica R7200 can be associated with a stronger interaction of this filler with the matrix.

The hardness behavior is shown in Fig. 8d. Increase of the hardness values must be associated with the increased modulus and the presence of hard silica domains. An additional factor can be partial migration of silica towards the surface [35]. When silica loading reaches about $4-5$ wt.- $\%$ further changes becomes partly suppressed showing worsening of phase compatibility.

\section{Conclusions}

A comprehensive study of model monomer/silica systems was carried out to find mutual interdependence of parameters characterizing these systems before, during and after curing. The decisive parameter proved to be stability of the silica dispersion associated with the formation of a solvation cell which during curing is converted into the interphase layer. Although the influence of monomer (polymer)/filler compatibility on many aspects of composite formation and properties was discussed in a number of papers, our results show how sensitive to this feature of the initial formulation are curing kinetics and composite properties. The investigated parameters: the Zeta potential, glass temperature, polymerization rate and conversion, glass transition, surface roughness, tensile properties and hardness evolve with the filler loading but show a maximum or a change in trend at the same composition. Thus, these parameters are strongly coupled. In the case of systems investigated in this work the threshold (optimum) composition is that containing about 5 wt.- $\%$ of silica. At this filler content dispersion stability is the greatest, polymerization the fastest, glass temperature the highest, and surface roughness and elongation to break show maxima. The results are qualitatively the same for 
compositions containing two types of silica and small quantitative differences only confirm the correctness of the conclusions. Although our article presents results only for systems based on one monomer, we observed also analogous dependences for other ethylene glycol dimethacrylates. Awareness of the existence of such a correlation can be helpful in design of the compositions and conditions for composite production.

Acknowledgments This work was supported by the Research Projects of Poznan University of Technology 03/32/DSPB/0604. Hubert Gojzewski acknowledges the Polish Ministry of Science and Higher Education for the project "Mobilnosc Plus" and the stipend "Stypendium dla wybitnych mlodych naukowcow".

Open Access This article is distributed under the terms of the Creative Commons Attribution 4.0 International License (http:// creativecommons.org/licenses/by/4.0/), which permits unrestricted use, distribution, and reproduction in any medium, provided you give appropriate credit to the original author(s) and the source, provide a link to the Creative Commons license, and indicate if changes were made.

\section{References}

1. Mohseni M, Bastani S, Jannesari A (2014) Influence of silane structure on curing behavior and surface properties of sol-gel based UV-curable organic-inorganic hybrid coatings. Prog Org Coat 77:1191-1199

2. Hajji P, David L, Gerard J-F, Pascault J-P, Vigier G (1999) Synthesis, structure and morphology of polymer-silica hybrid nanocomposites based on hydroxyethyl methacrylate. J Polym Sci B Polym Phys 37:3172-3187

3. Andrzejewska E (2001) Photopolymerization kinetics of multifunctional monomers. Prog Polym Sci 26:605-665

4. Schwalm R (2006) UV coatings, basisc, recent developments and new applications Elsevier Science, ISBN-10: 0444529799

5. Wang Y-Y, Hsieh T-E (2007) Effect of UV curing on electrical properties of a UV-curable copolyacrylate/silica nanocomposite as a transparent encapsulation resin for device packaging. Macromol Chem Phys 208:2396-2402

6. Güngör A, Kayaman-Apohan N, Mert A, Kahraman M-V (2008) Preparation and characterization of light curable hybrid coating: its potential application for dental restorative material. J Polym Res 15: 389-395

7. Xiao X, Hao C (2010) Preparation of waterborne epoxy acrylate/ silica sol hybrid materials and study of their UV curing behavior. Colloids Surf A Physicochem Eng Asp 359:82-87

8. Sadej M, Andrzejewska E, Kurc B, Gojzewski H, Jesionowski T (2014) Surface-dependent effect of functional silica fillers on photocuring kinetics of hydrogel materials. J Polym Sci A Polym Chem 52:3472-3487

9. Kaddami H, Pascault J-P, Gerard J-F (2004) Influence of the initiation rate on the polymerization kinetics of hydroxy ethyl methacrylate (HEMA) filled with HEMA-grafted silica preformed nanoparticles. Polym Eng Sci 44:1231-1239

10. Sow C, Riedl B, Blanchet P (2010) Kinetic studies of UVwaterborne nanocomposite formulations with nanoalumina and nanosilica. Prog Org Coat 67:188-194

11. Li F, Zhou S, You B, Wu L (2006) Kinetic investigations on the UVinduced photopolymerization of nanocomposites by FTIR spectroscopy. J Appl Polym Sci 99:1429-1436
12. Cho J-D, Kim Y-B, Ju H-T, Hong J-W (2005) The effects of silica nanoparticles on the photocuring behaviors of UV-curable polyester acrylate-based coating systems. Macromol Res 13:362-365

13. Sadej-Bajerlain M, Gojzewski H, Andrzejewska E (2011) Monomer/modified nanosilica systems: photopolymerization kinetics and composite characterization. Polymer 52:1495-1503

14. Andrzejewska E, Marcinkowska A, Podgórska M, Stępniak I, Sądej M (2009) Photopolymerization. New investigations. New materials. Polimery 54:327-333

15. Zou H, Wu S, Shen J (2008) Polymer/silica nanocomposites : preparation, characterization, properties and applications. Chem Rev 108:3893-3957

16. Bauer F, Flyunt R, Czihal K, Ernst H, Naumov S, Buchmeiser M-R (2007) UV curing of nanoparticle reinforced acrylates. Nucl Inst Methods Phys Res B Beam Interact Mater Atoms 265:87-91

17. Samuel S-P, Li S, Mukherjee I (2009) Mechanical properties of experimental dental composites containing a combination of mesoporous and nonporous spherical silica as fillers. Dent Mater 25:296-301

18. Mammeri F, Rozes L, Le Bourhis E, Sanchez C (2006) Elaboration and mechanical characterization of nanocomposites thin films. J Eur Ceram Soc 26:267-272

19. Karger-Kocsis J, Fakirov S (2009) Nano- and mircro- mechanics of polymer blends and composites. Hanser Pub, Munich. ISBN 978-156990-435-0

20. AEROSIL - Fumed Silica Technical Overview (2015) Evonik Industries 12

21. Perevyazko I, Vollrath A, Hornig S, Pavlov G-M, Schubert U-S (2010) Characterization of poly(methyl methacrylate) nanoparticles prepared by nanoprecipitation using analytical ultracentrifugation, dynamic light scattering, and scanning electron microscopy. J Polym Sci A Polym Chem 48:3924-3931

22. Andrzejewska E, Marcinkowska A (2008) New aspects of viscosity effects on the photopolymerization kinetics of the 2, 2-bis [4-(2hydroxymethacryloxypropoxy) phenyl] propane/triethylene glycol dimethacrylate monomer system. J Appl Polym Sci 110:2780-2786

23. Marcinkowska A, Andrzejewska E (2010) Viscosity effects in the photopolymerization of two-monomer systems. J Appl Polym Sci 116:280-287

24. Raghavan S-R, Carolina N (2000) Rheology of silica dispersions in organic liquids: new evidence for solvation forces dictated by hydrogen bonding. Langmuir 16:7920-7930

25. Van Der Hoeven PC, Lyklema J (1992) Electrostatic stabilization in non-aqueous media. Adv Colloid Interf Sci 42:205-277

26. Pugh R-J, Matsunaga T-T, Fowkes F-M (1983) Dispersibility and stability of carbon black in media of low dielectric constant. 1 . Electrostatic and steric contributions to colloidal stability. Colloids Surf 7:183-207

27. Spange S, Simon F, Heublein G, Jacobasch H-J, Börner M (1991) Donator acceptor behavior of aerosil in several organic liquids. Colloid Polym Sci 269:173-178

28. Morrison I-D (1993) Electrical charges in nonaqueous media. Colloids Surf A Physicochem Eng Asp 71:1-37

29. Cho J-D, Ju H-T, Hong J-W (2005) Photocuring kinetics of UVinitiated free-radical photopolymerizations with and without silica nanoparticles. J Polym Sci A Polym Chem 43:658-670

30. Andrzejewska E, Sadej M (2008) Photocurable methacrylate/ nanosilica composites. Polimery 53:70-72

31. Natarajan B, Li Y, Deng H, Brinson L-C, Schadler L-S (2013) Effect of interfacial energetics on dispersion and glass transition temperature in polymer nanocomposites. Macromolecules 46: 2833-2841

32. Qiao R, Deng H, Putz K-W, Brinson L-C (2011) Effect of particle agglomeration and interphase on the glass transition temperature of polymer nanocomposites. J Polym Sci B Polym Phys 49:740-748 
33. Ramanathan T, Stankovich S, Dikin D, Liu H, Shen H, Nguyen S-T, Brinson L-C (2007) Graphitic nanofillers in PMMA nanocomposites - an investigation of particle size and dispersion and their influence on nanocomposite properties. J Polym Sci B Polym Phys 45:2097-2112

34. Aso O, Eguiazábal J-I, Nazábal J (2007) The influence of surface modification on the structure and properties of a nanosilica filled thermoplastic elastomer. Compos Sci Technol 67:2854-2863

35. Amerio E, Sangermano M, Malucelli G, Priola A, Voit B (2005) Preparation and characterization of hybrid nanocomposite coatings by photopolymerization and sol-gel process. Polymer 46: 11241-11246 